\title{
Multi-Domain Fourier-Continuation/WENO Hybrid Solver for Conservation Laws
}

\author{
Khosro Shahbazi $^{*, a}$ Nathan Albin ${ }^{\mathrm{b}}$ Oscar P. Bruno ${ }^{\mathrm{b}}$ \\ Jan S. Hesthaven ${ }^{\text {a }}$ \\ ${ }^{a}$ Division of Applied Mathematics, Brown University, 182 George Street, \\ Providence, RI 02912, United States \\ ${ }^{\mathrm{b}}$ Applied $\&$ Computational Mathematics, California Institute of Technology, MC \\ 217-50, Pasadena , CA 91125
}

\begin{abstract}
We introduce a multi-domain Fourier-Continuation/WENO hybrid method (FCWENO) that enables high-order and non-oscillatory solution of systems of nonlinear conservation laws, and which enjoys essentially dispersionless, spectral character away from shocks, as well as mild CFL constraints. The hybrid scheme employs the expensive, shock-capturing WENO method in small regions containing discontinuities and the efficient FC method in the rest of the computational domain, yielding a highly effective overall scheme for applications with a mix of discontinuities and complex smooth structures. The accuracy, stability and efficiency of the new FC-based method for conservation laws is investigated for both problems in which solutions are smooth and problems for which solutions contain shock discontinuities; in particular, in the latter case we compare the efficiency of the hybrid FC-WENO method to that of a purely WENO-based approach. In a variety of examples, including an Euler problem that governs the interaction of a strong shock with a very small entropy wave, we show that the hybrid strategy is several times faster than the pure WENO solver for a comparable level of accuracy.
\end{abstract}

Key words:

Fourier continuation Methods, high-order WENO methods, multi-resolution methods, conservation laws, shock waves

* Corresponding author.

Email addresses: shahbazi@brown.edu (Khosro Shahbazi), nathan.albin@caltech.edu (Nathan Albin), bruno@caltech.edu (Oscar P. Bruno), jan.hesthaven@brown.edu (Jan S. Hesthaven). 


\section{Introduction}

Solutions of flow problems are often characterized by a challenging combination of sharp gradients, discontinuities and regions of complex but smooth flow structures; an example of such situations is provided by the well known shockinduced multi-material flow instabilities and the ensuing turbulent flows. To accurately capture all regimes in such complicated flow structures it is necessary to account adequately for both sharp shock discontinuities (without introduction of oscillatory behavior near shocks) as well as complex smooth flow structures. A well-known highly effective approach for the solution of the compressible Navier-Stokes equations governing such flows is based on a highorder weighted essentially non-oscillatory (WENO) finite difference method; in particular, high-order WENO algorithms have been used to produce successful simulations of the Rayleigh-Taylor instability [19] and Richtmyer-Meshkov instability $[20,21]$ in two and three space dimensions.

Unfortunately, the WENO finite difference methods give rise to a much higher computational cost than either the classical finite difference method or the Fourier spectral method - owing, mainly, to certain costly WENO operations: the characteristic decomposition of fluxes and the calculation of nonlinear weights for smooth and non-smooth flux contributions. Given the overall complexity required by WENO solutions of the unsteady Navier-Stokes equations in three space dimensions, it is essential to strive for more efficient solution strategies.

For the types of applications under consideration, the solution discontinuities are mainly local phenomena in both space and time, which suggests that a hybrid scheme could advantageously be used whereby the "expensive" WENO procedure is only used in parts of the space-time domain containing discontinuities, while a less costly, high-order numerical method is employed for the portion of the domain containing complex but smooth features. Such an approach requires use of a multi-domain formulation in which, at regular discrete time intervals, a discontinuity-sensing procedure is used to identify the sub-domains with either smooth or discontinuous content and adjust the computational solution approach accordingly.

The development of such a hybrid approach can be based on use of an efficient numerical method for smooth regions together with an effective "smoothnessindicator" strategy for identification of areas of smoothness and discontinuity. For the former, the classical central difference (upwind-biased or unbiased) and the Chebyshev-based spectral methods are both natural choices. To accurately identify discontinuities, on the other hand, the multi-resolution algorithm of Harten [7], which is based on differences of the point-values of a function and its high-order interpolated function on coarser grids has been shown to 
perform well $[8,9]$. In fact, in the contributions $[8,9]$ a hybridization of spectral Chebyshev and WENO methods for conservation laws in one and two space dimensions is proposed and applied to a complex simulation of early-stage two-dimensional Richtmyer-Meshkov instability. The preference of Chebyshev spectral methods over the central difference methods lies in the possibility of achieving higher order of accuracy near the non-periodic boundaries and solutions free of dispersion error (also known as pollution error). Absence of dispersion error is crucial for efficient simulation of problems with a wide range of spatial and temporal scales including transitional and turbulent flows [23].

An unfortunate consequence of the use of the Chebyshev spectral method is the presence of grid points that cluster near the boundaries of each such interval or sub-domain. On the other hand, the WENO finite difference method is defined on an equidistant grid, and thus the two underlying grids in a ChebyshevWENO hybrid do not conform in any natural manner. This implies that extra interpolation operations are required for transfer of data at overlapping regions between adjacent domains with two different discretization schemes, on one hand, and for the purpose of multi-resolution analysis, on the other. Further, for explicit time integration strategies, since the minimum grid spacing for the Chebyshev-Gauss-Lobatto points is $\mathcal{O}\left(1 / N^{2}\right)$, where $N$ is the number of grid points along a one dimensional mesh, the hybrid Chebyshev-WENO methods requires a much smaller $\left(\mathcal{O}\left(1 / N^{2}\right)\right)$ time step than a scheme based on an equidistant grid.

In the present work we propose an alternative approach in which we hybridize the WENO method with a recently proposed Fourier continuation (FC) method $[1,4,5]$. (Here we restrict our considerations to one dimensional problems. In view of the contributions $[1,4,5]$, which concern $\mathrm{FC}$ methods for PDEs in two and three spatial dimensions, however, we expect our FC-WENO approach should be applicable and, indeed, highly competitive for systems of conservation laws in both two- and three-dimensional space.) The FC approximation is based on a high-order periodic continuation of a (possibly nonperiodic) function, yet being a Fourier method, the FC approximation retains the equi-spaced grid points, has no dispersion (pollution) error, and, owing to its reliance on the Fast Fourier Transform, it is highly efficient. Further, in view of the FC method's use of equi-spaced grids, the interface with the WENO approach is much simpler and more effective than the one associated with the Chebyshev-WENO approach.

The remainder of the paper is organized as follows. In Sec. 2 we describe the FC method for approximation of functions, and in Sec. 3 we introduce our multidomain FC-WENO hybrid method for conservation laws in one-dimensional space. In Sec. 4 we then present a variety of numerical results for problems governed by the advection equation, the inviscid Burgers' equation and the Euler system in one space dimension. In this section we thus demonstrate the 
accuracy and stability of the FC method in the context of advection problems with smooth solutions in both single-domain and multi-domain formulations, and we assess the efficiency of our method, in terms of accuracy and speed, by comparing it with the pure WENO method and other related methodologies, in the context of challenging computational problems-including nonlinear systems of conservation laws with discontinuous solutions. In Sec. 5, finally, we present a few concluding remarks.

\section{The Fourier Continuation approximation}

Given a (typically non-periodic) function $f$ over the interval $[0,1]$ and given values of $f$ at an $N$-point equi-spaced grid $\left\{x_{k}\right\}_{k=1}^{N} \subseteq[0,1]$, the Fourier Continuation (Extension) method seeks to produce, on the basis of the given values $\left\{f\left(x_{k}\right)\right\}_{k=1}^{N}$, a periodic function $\tilde{f}$ defined over a larger domain $[0,1+d]$ that is defined by a finite number $M$ of Fourier modes,

$$
\tilde{f}(x)=\sum_{j \in g(M)} \hat{a}_{j} e^{\left(\frac{2 \pi i}{1+d} j x\right)}
$$

and that matches closely the original function $f$ throughout the original interval $[0,1]$. Here $g(M)=\{j \in \mathbb{N} \mid-M / 2+1 \leq j \leq M / 2\}$ for $M$ even and $g(M)=\{j \in \mathbb{N} \mid-(M-1) / 2 \leq j \leq(M-1) / 2\}$ for $M$ odd. In general, $N$ should be chosen larger than $M$ due to the intrinsic ill-conditioning of the resulting linear system; as shown in [2,3], the least-squares solution via singular value decomposition of the overdetermined system

$$
\tilde{f}\left(x_{k}\right)=\sum_{j \in g(M)} \hat{a}_{j} e^{\left(\frac{2 \pi i}{1+d} j x_{k}\right)} \quad \forall k=1, \cdots, N
$$

for the coefficients $\hat{a}_{j}$ leads to an effective Fourier Continuation strategy.

As an alternative method for eliminating the ill-conditioning without incurring the high $\mathcal{O}\left(N^{3}\right)$ computational cost that arises from the evaluation of the singular value decomposition [6] mentioned above (which, in application to solution of PDEs is prohibitively expensive), the contribution [4] proposes the following strategy: at first the restriction of the function $f$ to certain very small subintervals of $[0,1]$ containing the endpoints 0 and 1 (boundary intervals) are projected onto a space of discrete orthogonal polynomials (Gram polynomials $([25]))$ of small degree, and Fourier continuation of orthogonal bases of these spaces are precomputed using fine submeshes. The continuation of the original function then proceeds, at FFT speeds, using the projection coefficients of the function at the boundary segments.

In detail, let the left and right boundary segments of the original interval $[0,1]$, 
of equal size $\Delta$, be denoted by $\Delta^{\text {left }}$ and $\Delta^{\text {right }}$. Moreover, let the corresponding orthogonal (Gram) polynomial basis of degree $\leq p$ be based on a number $\gamma$ of equi-spaced grid points in $\Delta^{\text {right }}$ and $\Delta^{\text {left }}$ be $\phi_{l}^{\text {left }}$ and $\phi_{l}^{\text {right }}, l=0, \cdots, p$. Using the boundary intervals $\Delta^{\text {left }}$ and $\Delta^{\text {right }}$ (instead of the entire interval) we seek periodic extensions of the basis over the interval $[1-\Delta, 1+\Delta+2 d]$ (with periodicity of $2 \Delta+2 d$ ) such that the extensions interpolate the basis functions over $\Delta^{\text {right }}$ and $1+d+\Delta$ at a sufficiently large number of equi-spaced grid points. In other words, we seek functions $\tilde{\psi}_{\ell}(x)$ of the form

$$
\tilde{\psi}_{\ell}(x)=\sum_{j \in m(g)} \hat{a}_{j}^{\ell} e^{\left(\frac{\pi i}{\Delta+d} j x\right)}
$$

where the coefficients $\hat{a}_{j}^{\ell}$ are found by requiring, in the least squares sense, that

$$
\tilde{\psi}_{\ell}\left(y_{k}\right)=\sum_{j \in m(g)} \hat{a}_{j}^{\ell} e^{\left(\frac{\pi i}{\Delta+d} j y_{k}\right)}=\phi_{\ell}^{\mathrm{right}}\left(y_{k}\right)
$$

and

$$
\tilde{\psi}_{\ell}\left(y_{k}+\Delta+d\right)=\sum_{j \in m(g)} \hat{a}_{j}^{\ell} e^{\left(\frac{\pi i}{\Delta+d} j\left(y_{k}+\Delta+d\right)\right)}=\phi_{\ell}^{\text {left }}\left(y_{k}+\Delta+d\right)
$$

with

$$
y_{k}=1-\Delta+\frac{k \Delta}{\gamma-1} \quad k=0, \cdots, Q-1
$$

for an adequately large value of $Q$. Setting $\phi_{\ell}^{\text {right }}\left(y_{k}\right)=\phi_{\ell}^{\text {left }}\left(y_{k}+\Delta+d\right)$, $\ell=0, \cdots, p$ and $k=0, \cdots, Q-1$, for periodicity of the function in $[0,1+d]$, along with the uniqueness of the continuation dictate that all odd modes be zero. The Fourier extension of the basis functions then reduces to

$$
\tilde{\psi}_{\ell}(x)=\sum_{j \in m(g)} \hat{a}_{j}^{\ell} e^{\left(\frac{\pi i}{\Delta+d} j x\right)}, \quad j \text { even, }
$$

where the coefficients $\hat{a}_{j}$ are obtained by solving the overdetermined linear system

$$
\tilde{\psi}_{\ell}\left(y_{k}\right)=\sum_{j \in m(g)} \hat{a}_{j}^{\ell} e^{\left(\frac{\pi i}{\Delta+d} j y_{k}\right)}=\phi_{l}^{r}\left(y_{k}\right) \quad j \text { even }
$$

(As noted in [4], this calculation should be performed in high-precision arithmetic, and the small number of associated coefficients should be stored for use as part of the any general domain PDE solver.) To include the odd modes in the continuation of the basis function we consider functions $\tilde{\zeta}_{\ell}(x)$ such that they approximate, in the least squares sense, $\phi_{\ell}(x)$ and $-\phi_{\ell}(x+\Delta+d)$ in $\Delta^{\text {right }}$ and $1+d+\Delta^{\text {left }}$, respectively:

$$
\tilde{\zeta}_{\ell}\left(y_{k}\right)=\sum_{j \in m(g)} \hat{b}_{j}^{\ell} e^{\left(\frac{\pi i}{\Delta+d} j y_{k}\right)}=\phi_{\ell}^{\text {right }}\left(y_{k}\right)
$$

and

$$
\tilde{\zeta}_{\ell}\left(y_{k}+\Delta+d\right)=-\sum_{j \in m(g)} \hat{b}_{j}^{\ell} e^{\left(\frac{\pi i}{(\Delta+d)} j\left(y_{k}+\Delta+d\right)\right)}=-\phi_{\ell}^{\text {left }}\left(y_{k}+\Delta+d\right) .
$$


In this case all the even modes are zero and the Fourier extensions become

$$
\tilde{\zeta}_{\ell}(x)=\sum_{j \in m(g)} \hat{b}_{j}^{\ell} e^{\left(\frac{\pi i}{\Delta+d} j x\right)}, \quad j \text { odd }
$$

where the coefficients $\hat{b}_{j}^{\ell}$ are obtained by solving the linear system

$$
\tilde{\zeta}_{\ell}\left(y_{k}\right)=\sum_{j \in m(g)} \hat{b}_{j}^{\ell} e^{\left(\frac{\pi i}{\Delta+d} j y_{k}\right)}=\phi_{\ell}^{\text {right }}\left(y_{k}\right) \quad j \text { odd } .
$$

Having computed $\psi_{\ell}$ and $\zeta_{\ell}$ through Eqs. (7), (8), (11) and (12), we can now define the Fourier continuation approximation of the original function as follows: using the discrete inner products

$$
\begin{aligned}
\hat{f}_{\ell}^{\text {right }} & =\left(f, \phi_{\ell}^{\text {right }}\right), \\
\hat{f}_{\ell}^{\text {left }} & =\left(f, \phi_{\ell}^{\text {left }}\right),
\end{aligned}
$$

over $\gamma$ equi-spaced grid points in $\Delta^{\text {right }}$ and $\Delta^{\text {left }}$, respectively, the periodic extension $\tilde{f}$ of $f$ is obtained as

$$
\begin{aligned}
& \tilde{f}(x)=f(x) \quad 0 \leq x \leq 1 \\
& \tilde{f}(x)=\sum_{\ell}\left(\frac{\hat{f}_{\ell}^{\text {right }}+\hat{f}_{\ell}^{\text {left }}}{2}\right) \psi_{\ell}(x)+\sum_{\ell}\left(\frac{\hat{f}_{\ell}^{\text {right }}-\hat{f}_{\ell}^{\text {left }}}{2}\right) \tilde{\zeta}_{\ell}(x) \quad 1<x \leq 1+d .
\end{aligned}
$$

Remark 1. For our numerical examples we have taken the maximum polynomial degree as $p=5$, the number of boundary points $\gamma=p+1=6$, and $d / \Delta=25 / 5$. The extensions $\psi_{\ell}$ and $\zeta_{\ell}$ are computed using $Q=150$ and $g=63$. Note that these functions are computed only once and their values are stored at $x_{k}=1+k / 26, k=1, \cdots, 25$. These function values are then used for the computation of the extension of a general function through Eq. (14b).

Remark 2. It is interesting to note that, while the polynomial projection of the original function implies a finite order of convergence of the method, the Fourier continuation still retains the desirable property of having no pollution error (as demonstrated in Sec. 4): for a certain accuracy level, as the wave number of the solution increases, the required number of points per wave length remains constant.

\section{Multi-domain Fourier-Continuation/WENO hybrid method}

In the following we describe the three main elements of our multi-domain hybrid FC-WENO method for solution of conservation laws. Our method is based on use of the FC method for the smooth portions of the solutions, the 
WENO method for regions with steep gradients or discontinuous solutions, and the smoothness indicator, which allows us to determine when to switch between the two techniques. For the temporal discretization, we use a standard third-order total total variation diminishing (TVD) Runge-Kutta method [24].

\subsection{FC method for conservation laws with smooth solutions}

For simplicity, in this section we consider smooth solutions of a nonlinear conservation law of the form

$$
\frac{\partial u(t, x))}{\partial t}+\frac{\partial f(u(t, x))}{\partial x}=0
$$

defined on a one-dimensional spatial domain $\Omega=[a, b]$ in the time interval $[0, T]$, subject to appropriate initial and boundary conditions. Given a set of equi-spaced grid points, $x_{k}=k(b-a) /(N-1), k=0, \cdots N-1$, we seek $u_{h}\left(t, x_{k}\right)$, an approximation to $u\left(t, x_{k}\right)$, which satisfies the equation in a collocation sense

$$
\frac{\partial u_{h}\left(t, x_{k}\right)}{\partial t}+\frac{\partial u_{h}\left(t, x_{k}\right)}{\partial x} \frac{f\left(u_{h}\left(t, x_{k}\right)\right)}{\partial u}=0 \quad \forall k=0, \cdots, N-1 .
$$

Note that we have not expressed our equation in conservation form, since the form (16) of the equation leads to a more efficient smooth-region algorithm.

The spatial derivative $\frac{\partial u_{h}\left(t, x_{k}\right)}{\partial x}$ is computed using the Fourier continuation of the numerical solution. In view of equation (2) we have the Fourier continuation expansion of $u_{h}\left(t, x_{k}\right)$,

$$
u_{h}(t, x)=\sum_{j \in g(M)} \hat{a}_{j}(t) e^{\left(\frac{2 \pi i}{b+d-a} j x\right)}
$$

and the spatial derivative is computed as

$$
\frac{\partial u_{h}\left(t, x_{k}\right)}{\partial x}=\sum_{j \in g(M)} \frac{2 \pi i j}{(b+d-a)} \hat{a}_{j}(t) e^{\left(\frac{2 \pi i}{b+d-a} j x_{k}\right)} .
$$

As we observe in Sec. 4, this approach may be weakly unstable when we apply Dirichlet boundary conditions or domain splitting. To remedy this, we use a very weak exponential filter to damp out high-frequency modes and thus stabilize the numerical method with minimal adverse impact on accuracy [16]. Specifically, after computing the Fourier coefficients $\hat{a}_{j}(t)$ on the extended domain, we compute the modified coefficients $\tilde{a}_{j}(t)$, as

$$
\tilde{a}_{j}(t)=\exp \left(-\beta(|j| / N / 2)^{2 q}\right) \hat{a}_{j}(t)
$$


leading to a modified version of (18)

$$
\frac{\partial u_{h}\left(t, x_{k}\right)}{\partial x}=\sum_{j \in g(M)} \frac{2 \pi i j}{b+d-a} \tilde{a}_{j}(t) e^{\left(\frac{2 \pi i}{b+d-a} x_{k} j\right)} .
$$

In (19), we choose $\beta$ such that the highest mode, $\tilde{a}_{N / 2}(t)$, vanishes that is, $\beta=-\log \left(\varepsilon_{M}\right)$, where $\varepsilon_{M}$ is the machine precision, typically $10^{-16}$. In this work, the filter order, $q$, is typically chosen $q \approx N / 2$ for $N<200$. For larger $N>200$, the order of the filter is required to be lowered, $q<N / 2$, to maintain stability. The impact of such a weak filter is minimal, as is demonstrated clearly by the numerical examples discussed later in this text.

Remark 3. For nonlinear problems, the use of exponential filter serves the additional purpose of removing energy accumulated at high frequencies, and thus stabilizing the calculations by controlling aliasing errors.

Remark 4. Note that with $p=5$ in the FC approximation, the derivative computation is technically fifth-order accurate, i.e., our FC method for the conservation law (15) with smooth solution is formally fifth-order accurate.

Remark 5. Regarding the implementation of the derivative calculations, the Fourier coefficients in (17) are first computed using a fast Fourier transform (FFT) and point-values of the derivatives in (20) are obtained using an inverse FFT. Both steps, FFT and inverse FFT, can be computed with a complexity of $\mathcal{O}(N \log (N))$. Alternatively, the derivative calculation can be performed using a matrix-vector product with a cost of $\mathcal{O}\left(N^{2}\right)$, where the matrix representing the effect of differentiation and the vector being the collocation values of the function [17]. For larger values $N$, the former is clearly preferred and that is the approach we consider here.

Remark 6. Equation (16) is in non-conservative form. The equivalent conservative form (for smooth problem) is

$$
\frac{\partial u_{h}\left(t, x_{k}\right)}{\partial t}+\frac{\partial f\left(u_{h}\left(t, x_{k}\right)\right)}{\partial x}=0 \quad \forall k=0, \cdots, N-1 .
$$

In all our numerical tests, both conservative and non-conservative $\mathrm{FC}$ discretization of the conservation laws yield almost identical results. Our preference for the non-conservative form is guided by efficiency. While the nonconservative form requires only the continuation of $u$ and its filtering, the conservative form requires continuation and filtering of both $u$ and the flux, $f(u)$ and, thus, renders the scheme more expensive.

Remark 7. In the multi-domain formulation of the FC method, the stability is achieved by considering overlapping domains. For the fifth-order FC method, we found empirically that an overlap of three grid points is sufficient for stability. 


\subsection{WENO Methods for conservation laws with discontinuous solutions}

WENO finite difference methods are finite difference schemes tailored to conservation laws involving discontinuous solutions - or, more generally, solutions which contain steep gradients. The details of the WENO schemes are welldocumented $[12,15]$, and we therefore give only a brief presentation of the essential elements of these methods.

A classical spatial finite difference discretization of (15) seeks point-wise enforcement of the equation at a set of equi-spaced grid points $x_{k}=k(b-$ a) $/(N-1), k=0, \cdots N-1$, as

$$
\frac{\left.\partial u_{h}\left(t, x_{k}\right)\right)}{\partial t}+\frac{\hat{f}\left(t, x_{k+1 / 2}\right)-\hat{f}\left(t, x_{k-1 / 2}\right)}{\Delta x}=0,
$$

where $\Delta x=x_{k}-x_{k-1}, u_{h}\left(t, x_{k}\right)$ is a numerical approximation of $u\left(t, x_{k}\right)$ and where $\hat{f}\left(t, x_{k+1 / 2}\right)$ is a numerical flux. The numerical flux (with shorthand notation $\left.\hat{f}_{k+1 / 2}\right)$ is defined using a numerical solution at $x_{k}$ and $r$ solutions to the left and $s$ solutions to the right of $x_{k}$ :

$$
\hat{f}_{k+1 / 2}=\hat{f}\left(u_{k-r}, \cdots, u_{k+s}\right) .
$$

Here $\hat{f}$ is required to be Lipschitz continuous in all arguments and consistent with the physical flux $f$, that is, $\hat{f}(u, \cdots, u)=f(u)$. Based on the LaxWendroff theorem, the solution of this conservative scheme, if it converges, converges to the weak solution of the original equation (15). The precise choice of the numerical flux $\hat{f}_{i+1 / 2}$ is obtained using a high-order WENO reconstruction procedure outlined below.

To guarantee entropy dissipation, the solution flux is first split into positive and negative parts using Lax-Friedrichs splitting as $f(u)=f^{+}(u)+f^{-}(u)$, with $f^{+}(u)=(f(u)+\alpha u) / 2, f^{-}(u)=(f(u)-\alpha u) / 2$, and $\alpha=\max _{u} \frac{d f(u)}{d u}$. The reconstruction is then applied for each positive and negative fluxes separately, before adding up to give the numerical flux.

A finite difference reconstruction of the flux, for instance for $f^{+}\left(u_{j+1 / 2}\right)$, can be computed using solutions in $q$ different stencils,

$$
\mathcal{S}_{r}(k)=x_{k-r}, \cdots, x_{k-r+q-1} \quad r=0, \cdots, q-1,
$$

as

$$
\hat{f}_{r}^{+}\left(u_{k+1 / 2}\right)=\sum_{n=0}^{q-1} C_{r, n} f^{+}\left(u_{k-r+n}\right) .
$$

The constant coefficients $C_{r, n}$ (given in [11] up to order seven) are chosen such that the approximate solution is accurate with to order $q$ in regions in 
which the exact solution is sufficiently smooth. On the other hand, if in a stencil, the solution or one of its $j$-derivatives $j \leq q$ contains a discontinuity, that stencil must be excluded from the flux approximation. This nonlinear procedure is referred to as an essentially non-oscillatory (ENO) schemes [14]. A slightly modified approach, offering computational advantages (see [11]), is to consider a convex combination of all computed fluxes $f_{r}^{+}\left(u_{j+1}\right)$ in $q$ stencils as

$$
f^{+}\left(u_{k+1 / 2}\right)=\sum_{r=0}^{p-1} w_{r} f_{r}^{+}\left(u_{k+1 / 2}\right),
$$

where $w_{r}$ is chosen such that the approximation is of order $2 q-1$ accuracy in cases where the solution is sufficiently smooth in all stencils. An explicit expression for $w_{r}$, based on a local smoothness indicator for orders $q=2$, and 3 , are given in [10] and for higher orders up to $q=6$ in [13].

Remark 8. For system of conservation laws, such as the Euler equations, it is essential to apply the WENO reconstruction to the fluxes in the eigenvector space. This is performed through multiplication of the flux vectors with the left eigensystem. The reconstructed fluxes are then transferred back to the conservative fluxes through multiplication with the right eigensystem [10].

\subsection{Detecting the discontinuities}

Detecting discontinuities in the solution or its derivatives is crucial for the hybrid strategy to be effective. For systems of nonlinear conservation laws like the Euler system of gas dynamics, the location of the discontinuities, e.g. shocks, are not known a priori and propagate as the solution advances. We therefore need to extract smoothness information from the solution field at regular intervals in the time integration procedure. To this end, the multiresolution (MR) analysis introduced by Harten [7] and later used in the context of a hybrid spectral-WENO method by Costa and Don [8] has proven quite effective. We present a brief description of this approach in what follows; a detailed account can be found in $[7,8]$.

Using the solution values $f_{k}^{0}$ at the set of equi-spaced grid points $x_{k}^{0}$ ( $k=$ $2, \cdots, 2 N+1)$ at which the discretized governing equations are solved, we construct the solution averages $f_{k}^{1}$ at one level coarser grid points $x_{k}^{1}$ with $k=1, \cdots, N$ as

$$
f_{k}^{1}=\frac{f_{2 k}^{0}+f_{2 k+1}^{0}}{2} \quad \forall k=1, \cdots, N .
$$

Let $p_{s}(x)$ denote the polynomial approximation of order $s$ interpolating $f_{k}^{1}$ at the coarser grid points $x_{k}^{1}$. The approximation differences, $d_{k}=f_{k}^{0}-p_{s}\left(x_{k}^{0}\right)$, have the property that if $f(x)$ has $r-1$ continuous derivatives and a jump 
discontinuity at $r$ th derivative, then

$$
d_{k}=\left\{\begin{aligned}
\Delta x^{\mathrm{right}}\left[\frac{d^{\mathrm{right}} f_{k}}{d x^{\mathrm{right}}}\right] & s \geq r, \\
\Delta x^{s} \frac{d^{s} f_{k}}{d x^{s}} & s<r,
\end{aligned}\right.
$$

where $\Delta x$ denotes the coarse grid spacing and where $[\cdot]$ denotes jumps across a discontinuity. This implies that the higher the smoothness of the solution, and the higher the order of the polynomial approximation on the coarser grid (in the case of smooth function) is, the smaller the approximation differences, $d_{k}$. We thus adopt a tolerance $\epsilon$, below which the function is assumed to be smooth and otherwise non-smooth. Once we have found the approximate locations of shocks by this method, the domains containing smooth solutions are treated by the FC method and those with non-smooth solutions are treated by the WENO scheme.

Remark 9. Note that for low resolution (i.e., for representations of the solution using a small number of spatial grid points) the multi-resolution analysis may fail to distinguish between a discontinuity and a large smooth solution gradient. An illustration of this fact is presented below in the case of the Euler system. In such regions, the algorithm assumes the solution to be non-smooth and it therefore evolves the solution by means of the WENO approach.

\section{Numerical Examples}

In this section we first present results produced by our FC algorithm for advection equations with smooth solutions, and we include comparisons with results produced by means of central difference (CD) methods. We then consider a number of results for the inviscid Burgers' equation, and finally, we present an extensive set of comparisons of our hybrid FC-WENO method with pure WENO for shock-entropy-wave-interaction problems. In the below FC-WENO hybrid calculations, for the multi-resolution analysis, we used the order of the interpolating polynomial on the coarse grid $s=9$, and the tolerence $\epsilon \approx 1.0^{-4}$.

\subsection{Advection equation}

We consider the advection equation, Eq. (15) with $f(u(t, x))=u(t, x)$, in a periodic spatial domain $[0,2 \pi]$ with a sinusoidal initial condition $u=\sin (\kappa x)$

and we solve the problem using three different methods: a fifth-order Fourier continuation (FC5) method, a fifth-order central difference (CD5) and a sixthorder central difference (CD6) method [26]. 


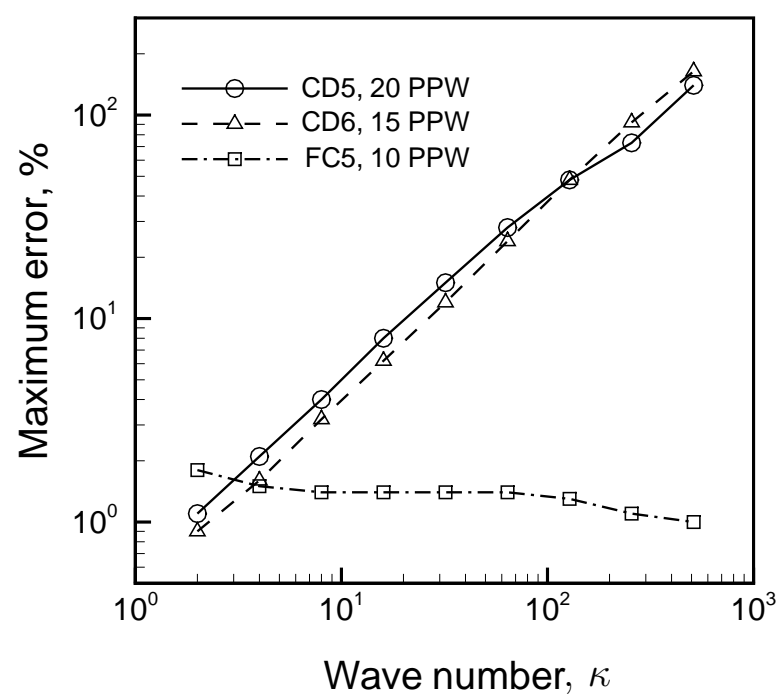

Fig. 1. Comparison of accuracies resulting from the fifth-order Fourier continuation (FC5) method and the central difference methods of orders 5 and 6 (CD5 and CD6, respectively) for the solution of the advection equation with a sinusoidal exact solution. The figure displays the maximum percent error (over all time steps to final time $T=100$ ) as a function of the wave-number for 20, 15 and 10 points per wavelength (PPW) for CD5, CD6 and FC5, respectively.

The results at the final time $T=100$ are compared in Fig. 1, which shows the maximum error over all time as a function of the wave number $\kappa$. For the FC5 method we assume 10 points per wave length (PPW) and CD5 and CD6 having 20 and 15 PPW, respectively. We observe that while error grows linearly with increasing wave number for the central difference methods, the situation is quite different for the FC method - for which the error remains constant at around 1\%. This illustrates the significant advantage provided by the FC method over central difference methods for multi-scale problems with wide frequency spectra. It is remarkable that due to the accumulation of phase error, the pollution error of the central difference method cannot be entirely eliminated by increasing the approximation order.

We next study the rate of convergence of the FC5 method for solving the advection equation in the spatial domain $[0,1]$ with the low-frequency exact solution $u(t, x)=\exp (\cos (x-t))$. In Fig. 2 the maximum error over all time steps up to the total time $T=100$ are plotted versus the grid spacing for the FC5 method and, for comparison, for the CD5 and CD6 methods as well. As expected, the FC5 method delivers approximately fifth-order accuracy. The performance of the FC method for solving the advection equation in the presence of Dirichlet boundary condition, with and without the use of the exponential filter, is shown in Figs. 3(a) and (b). In Fig. 3(a), the maximum error against time is plotted for filtered and unfiltered numerical solutions. While the unfiltered solution exhibits numerical instabilities characterized by 


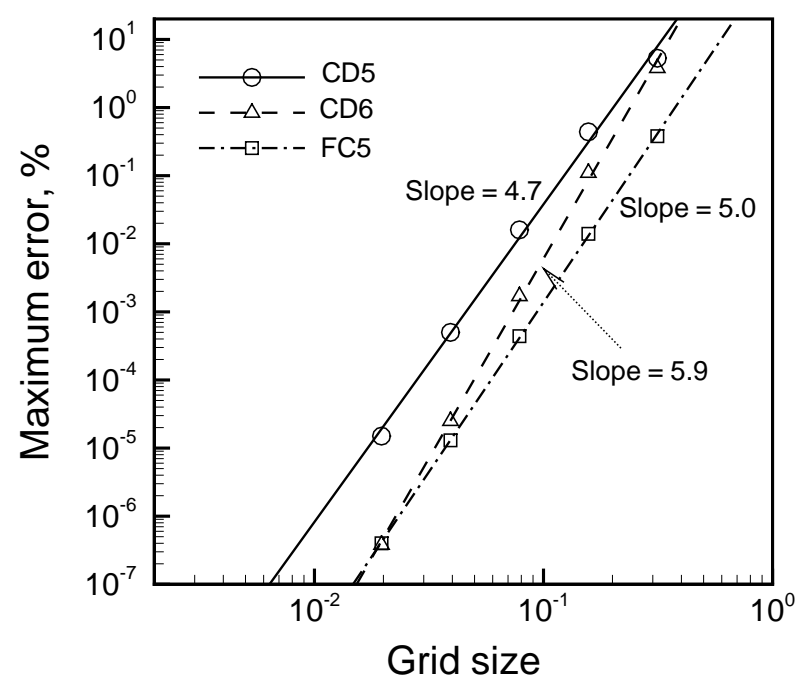

Fig. 2. Comparison of accuracies resulting from the fifth-order Fourier continuation (FC5) method and the central difference methods of order 5 and 6 (CD5 and CD6, respectively) for the solution of the advection equation with exact solution $u=\exp (\cos (x-t))$. The figure displays the maximum percent error (over all time steps to final time $T=100$ ) as a function of the spatial grid size. For each data set, the slope of the best curve fit is also shown.

rapid increase of the error, the filtered solution remains stable with small oscillations in error. Moreover, as seen in Fig. 3 (b), the filter does not have an adverse effect on the convergence rate, since the filtered solution exhibits a sixth-order average convergence rate. We note that an alternative to filtering that also achieves stability for the advection equation is the use of certain modifications to the FC method that are introduced in [1]. However, since we will be using the filter to control energy accumulation in the following treatment of nonlinear problems in any case, we do not make use of these modifications in the present work.

Finally, we demonstrate that the rate of convergence of the FC5 remains approximately unchanged for the multi-domain formulation. To this end, we solve the advection equation in $[0,2 \pi]$ with the exact solution $u(t, x)=\exp (6 \cos (x-$ $t)$ ) and periodic boundary condition using different numbers of domains $N D=$ $4,8,16$, and 32 and a fixed number of points per domains $N P=21$. The results for the total time $T=100$ are depicted in Figure 4, showing an average rate of convergence of 5.6. 


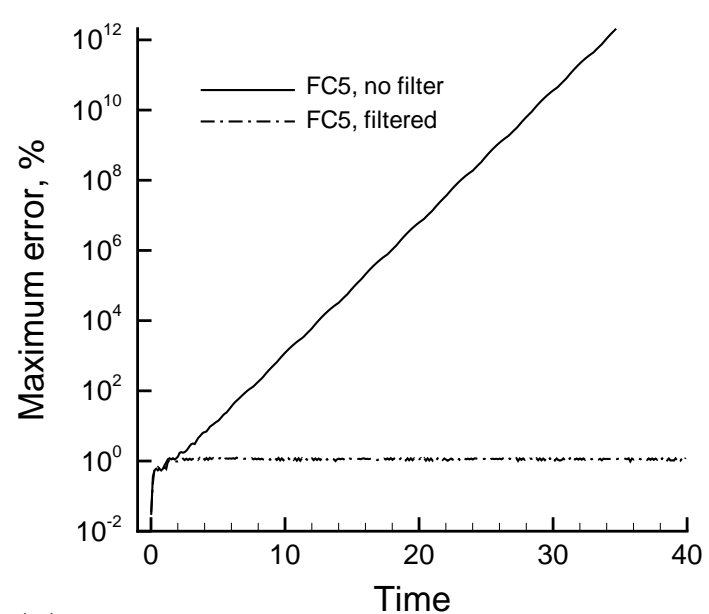

(a)

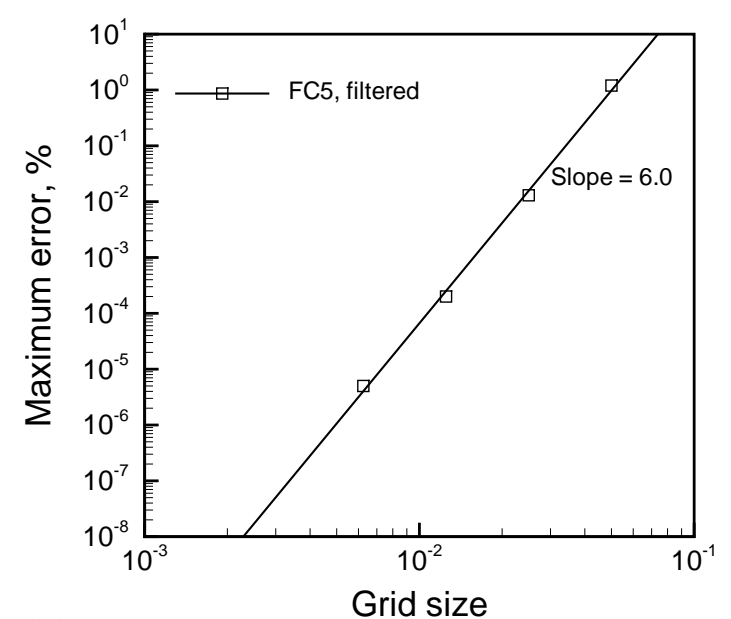

(b)

Fig. 3. (a) Stability of the fifth-order Fourier continuation (FC5) as applied to the solution of the Dirichlet problem for the advection equation considered in the text, in the interval $[0,1]$, and with exact solution given by $u=\exp (6 \cos (x-t))$. For this tests we have used $N P=21$ and we show results with and without the use of an exponential filter. (b) Convergence as a function of the grid size for the filtered FC5 method in the solution of the problem consider in the portion (a) of this figure. In (b), the best line fit to data and its slope are also shown.

\subsection{Burgers' equation}

We consider the inviscid Burgers' equation obtained by setting $f(u(t, x))=$ $u^{2}(t, x) / 2$ in (15). The computational domain is $[-1,1]$ with periodic boundary conditions and the initial condition is taken to be $u(0, x)=(1+\sin (\pi x)) / 2$. We first study the accuracy of the FC5 method for Burgers' equation using both single-domain and multi-domain formulations. In Fig. 5, the maximum error is plotted versus the grid spacing for the final time $T=0.25$, where the solution remains smooth. For the single-domain method, the number of points $N P=21,41,81$, and 161 , and for the multi-domain form $N P=21$ and the number of domains are $N D=1,2,4$, and 8 . As is clear from the figure, the single- and multi-domain variants leads to comparable accuracy levels and convergence rates of 5.5 and 5.3, respectively. (An improved multidomain strategy, by which these slight differences in accuracy are avoided, is presented in reference [1].)

We also consider Burgers' equation at the later time $T=0.75$, by which time a discontinuity in the solution has appeared, as produced by two different methods: the proposed hybrid FC-WENO method with fifth-order accuracy for both the FC and the WENO parts (FC5-WENO5), and a pure fifth-order WENO (WENO5) scheme. The computed solutions are shown in Figure 6(a), while the convergence rates are depicted in Figure 6(b). As is evident from the 


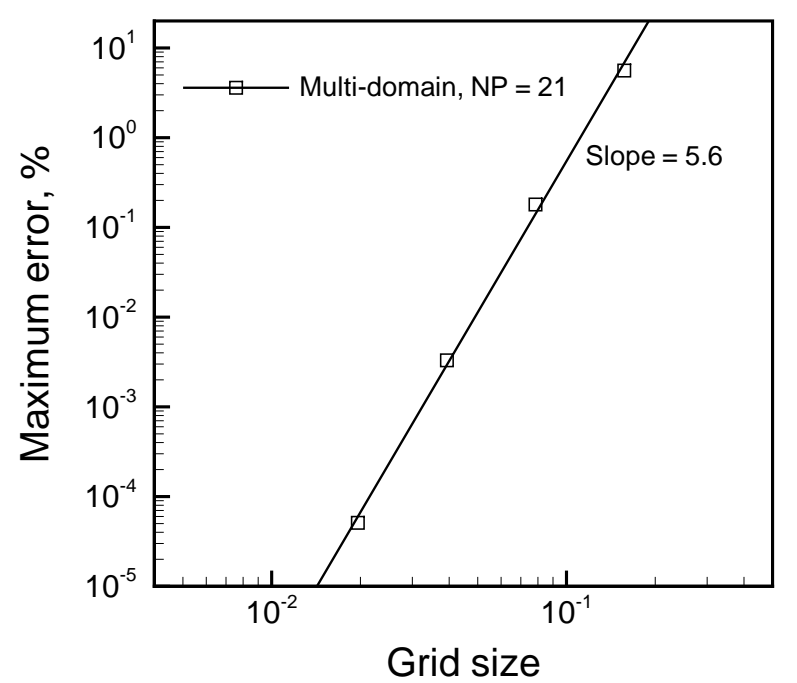

Fig. 4. Convergence of the fifth-order Fourier continuation (FC5) method as applied to the solution of the advection equation in the interval $[0,2 \pi]$ with an exact solution $u=\exp (6 \cos (x-t))$ and periodic boundary condition. The final time is $T=100$ and we use various number of domains $N D=4,8,16$, and 32 with $N P=21$ points each. The best line fit to data and its slope are also shown.

figures, both the hybrid FC5-WENO5 and the pure WENO5 methods yield almost identical solutions and convergence rates of 0.8 in the $L_{1}$ error norm.

\subsection{The Euler system}

The Euler equations in one-dimensional space are given by

$$
\frac{\partial \mathbf{u}}{\partial t}+\frac{\partial \mathbf{f}(\mathbf{u})}{\partial x}=0
$$

where $\mathbf{u}=[\rho, \rho v, \rho E]$ and $\mathbf{f}(\mathbf{u})=\left[\rho v, \rho v^{2}+p,(\rho E+p) v\right]$. Here, $\rho, v, E$ and $p$, which represent, respectively, the density, the velocity, the total energy and the pressure, are subjected to appropriate initial and boundary conditions.

We consider two test problems, both featuring the interaction of a Mach 3 shock with an entropy wave. The first problem (a shock/small-entropy-wave interaction case), features a small amplitude entropy wave, and allows for a quantitative comparison of our computed solutions with analytical results obtained using a linear analysis of the Euler equations. The second problem, (a shock/entropy-wave interaction configuration), involves an entropy wave with finite amplitude. No exact solution is known for this case. 


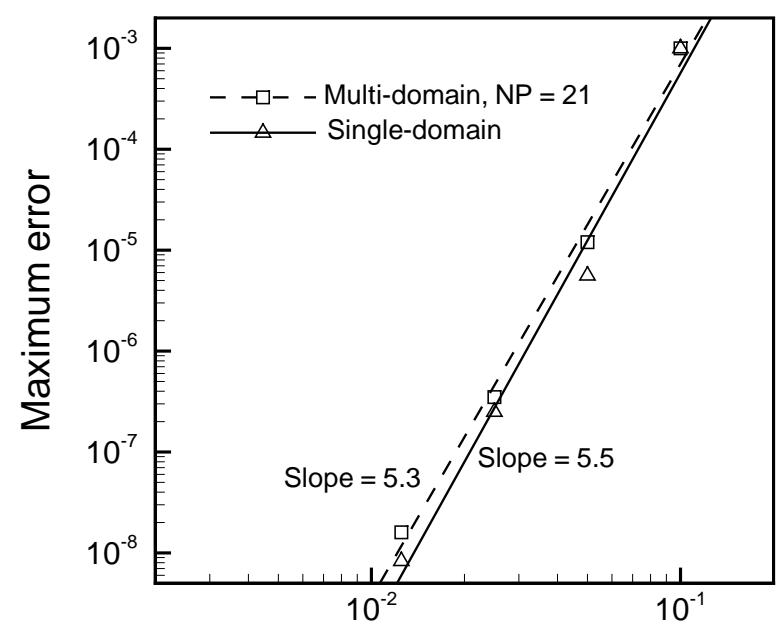

Grid size

Fig. 5. Convergence of the fifth-order Fourier continuation (FC5) method for the solution of the Burgers' equation in $[-1,1]$ with initial condition $u(0, x)=(1+\sin (\pi x)) / 2$. Results are obtained at the final time $T=0.25$ for both multi-domain and single domain formulations. For single-domain method, the number of points $N P=21,41,81$, and 161, and for the multi-domain $N P=21$ and the number of domains $N D=1,2,4$, and 8 . For each data set, the best curve fit and its slope are also shown.

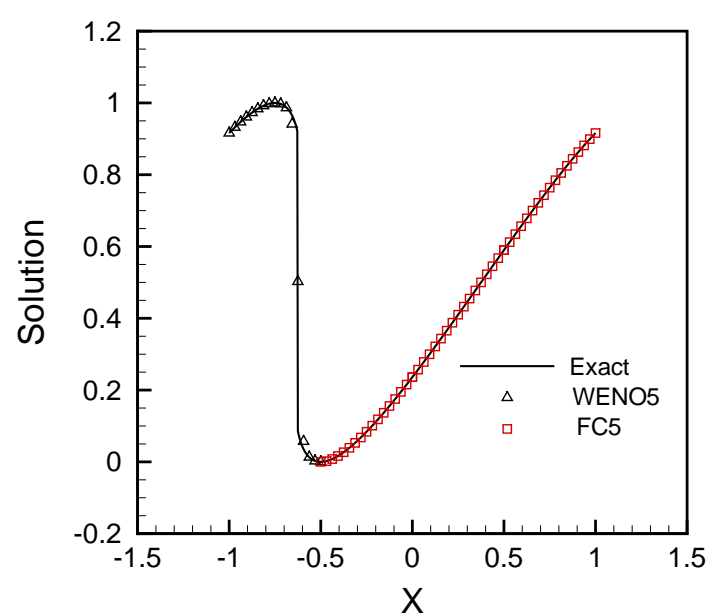

(a)

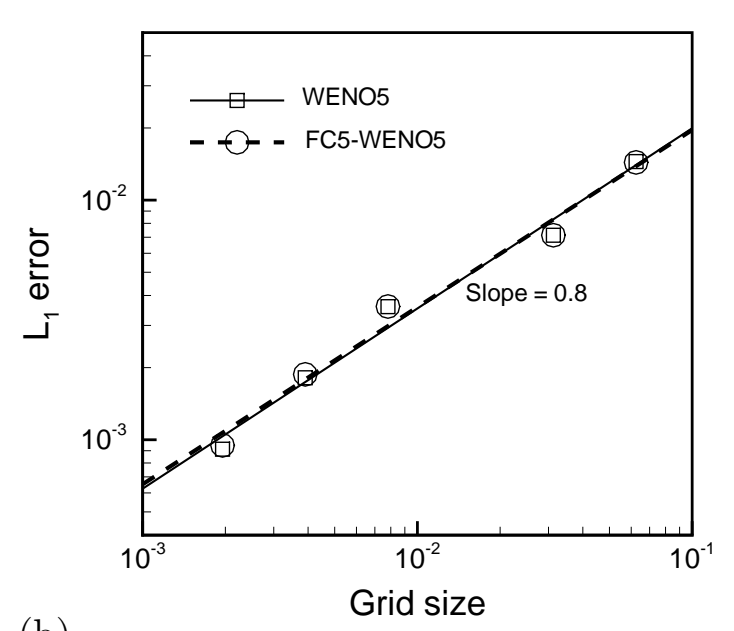

(b)

Fig. 6. (a) Discontinuous solution of Burgers' equation at $T=0.75$ computed using the hybrid FC5-WENO5 method; (b) Convergence of the pure WENO5 and hybrid FC5-WENO5 for the same test. In (b), the best curve fit to data and its slope are also shown.

\subsubsection{Shock/small-entropy-wave interaction test}

In order to quantify the performance of the hybrid FC-WENO methods we consider the interaction of a right-moving Mach 3 shock with a very small 
entropy wave. The spatial domain is $[-10,10]$ and the initial and boundary conditions are given by

$$
(\rho, v, p)(0, x)=\left\{\begin{aligned}
(3.857143,2.629369,10.33333) & x \leq-9.5 \\
(1.0,0.0,1.0) & -9.5 \leq x \leq-8.85 \\
(\exp (-0.01 \sin (13(x-8.85))), 0,1.0) & x>-8.85
\end{aligned}\right.
$$

The effect produced by a strong shock as it passes through a very small entropy wave is characterized by a sudden rise in the wave frequency and a sudden decline in the wave amplitude. These discontinuous changes in the frequency and amplitude, which are functions of the mean flow Mach number, can be obtained accurately, for sufficiently small entropy waves, through analysis of the linearized Euler equations [18] - thus allowing a quantitative assessment of the accuracy of our hybrid method.

We now present some quantitative results for solutions of the Euler system with the above initial conditions at the final time $T=5.0$, and for three different settings. Considering first a high-resolution (fine) calculation using the pure ninth-order WENO (WENO9) method with the total number of grid points $N P=10241$, we find that the computed entropy amplification for the fine calculation is within $0.1 \%$ of the amplification factor of 0.841037 produced by the linear analysis [18]. The fine result is thus considered exact up to this error tolerance. We then compare these values to those obtained by means of two low-resolution (coarse) calculations using the pure WENO5 method with $N P=2561$ and the hybrid FC5-WENO5 with $N P=33$ and the number of domains $N D=80$, hence an equivalent of total grid points of 2561 .

Figure 7 (a) depicts the computed entropies for the three methods and Fig. 7 (b) is a close-up view of the same data. As seen from Fig. 7 (b), right behind the shock $(x \approx 8.0)$, both coarse calculations yields results very close to that of the high-resolution computation. Away from the shock $(x \approx 4.0)$, in contrast, the computed entropy using the WENO5 method displays much larger dissipation as compared to that of the FC5-WENO5.

To overcome the excessive damping, we replaced the fifth-order WENO with a ninth-order WENO discretization for the coarse calculations and repeated the same test. The results are shown in Fig. 7 (c). Comparing the results in Fig. 7 (c) with those in Fig. 7 (b) clearly indicates that increasing the order of WENO scheme from five to nine yields more accurate entropy field in particular for the pure WENO method and improved agreement with the result of the hybrid scheme.

We next give a detailed quantitative comparison of the results produced by our hybrid FC-WENO solver with those resulting from the pure WENO method 


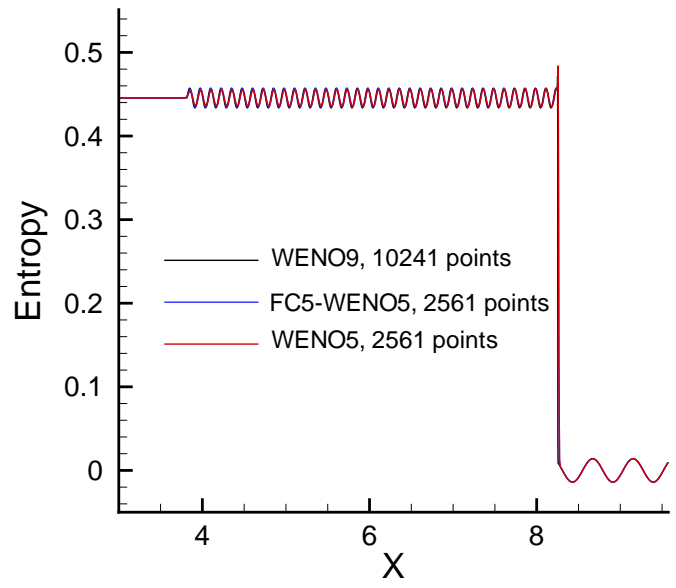

(a)

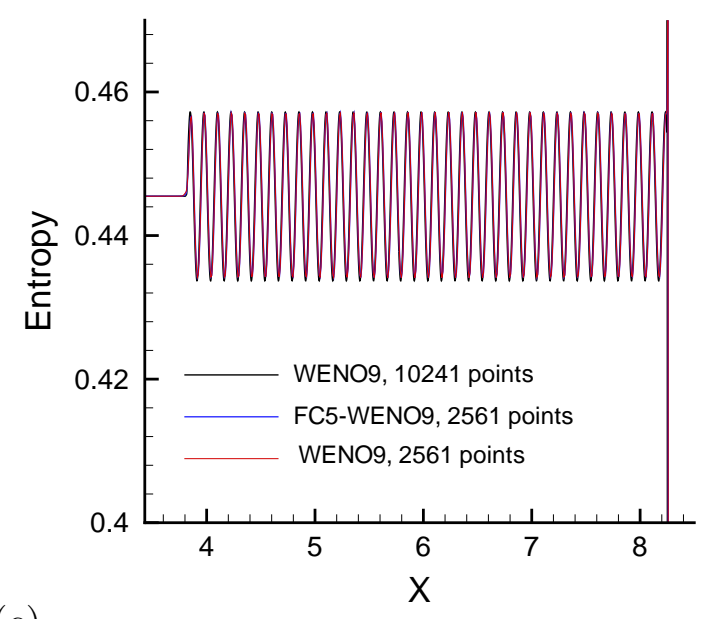

(c)

Fig. 7. Entropy profiles for shock-small-entropy-wave-interaction test at the total time $T=5.0$; (a) The entropy profile computed using the pure WENO9 with 10241 grid points - treated as the exact solution, and two coarse results obtained using a pure WENO5 with 2561 grid points and the hybrid FC5-WENO5 with $N P=33$ and $N D=80$, and, hence, the same total number of grid points. (b) close-up view of the same results, showing the inaccuracy of the WENO5 and FC5-WENO5 results; (c) close-up view of the entropy profiles obtained using the same setting as in (a) except for in the coarse calculations the fifth-order WENO is replaced by the ninth-order, demonstrating improved accuracy and agreement with the fine WENO9 results.

for the entropy amplification at two different Mach numbers. Tables 1 and 2 presents results for Mach three and Table 3 for Mach six. In the upper half of Table 1, the percent error in entropy amplification at two locations near to and far from the shock ( $x \approx 4.0$ and $x \approx 8.0$, respectively) and total CPU times (seconds) are given for the WENO5 and FC5-WENO5 methods. In the lower half the same quantities are listed for the WENO9 and FC5-WENO9 methods. For the hybrid method we chose $N D=80$ and $N P=33$, and for the pure 
WENO methods we use the equivalent total number of points $N P=2561$. The error calculations are based on the analytical entropy amplification factors obtained using linear analysis. These are 0.841037 and 0.68485 for Mach numbers three and six, respectively (see [18], Eq. (7)).

Several points should be noted in connection with Table 1. First, the ninthorder WENO method yields lower error than the fifth-order WENO version, as expected. Second, the hybrid schemes are faster than the corresponding pure WENO schemes, with a threefold advantage in the fifth-order case and a sixfold in the ninth-order case. Finally, using WENO9 instead of WENO5 in a hybrid scheme significantly improves the accuracy with a minor increase in total cost. This confirms that, indeed, it is highly advantageous to exploit the fact that only a small percentage of domains need to be treated by the WENO method in the hybrid scheme, and, further, it shows that it is beneficial to use WENO9 instead of WENO5 in the hybrid approach - since the increased cost of the WENO9 compared to WENO5 (which is approximately twofold) leads to a very small increase in the total cost of the hybrid algorithm. The actual number of WENO domains averaged over all time steps in the hybrid computations of this test problem is approximately two.

Table 2 reports data similar to that in Table 1, but with one level higher in the resolution: $N D=160$. As seen from the table, the increased resolution yields increased accuracy. For instance, the errors in the WENO9 and FC-WENO9 results drop below one percent. Similar to the former case, the hybrid approach appears several-fold faster than the pure WENO method.

Table 3, finally, presents data for a higher value of the Mach number, namely, Mach six, demonstrating similar trends as for the lower Mach number dataexcept for the fact that, in this case, the error values are uniformly larger than those presented in Table 2. This is expected since higher Mach numbers give rise to larger solution gradients, and hence require higher resolution for the same level of accuracy.

We also considered a different version of hybrid schemes in which a six-order central difference (CD6) method was combined with the WENO9 method. For this problem we did observe that at a comparable level of accuracy, this combination appears to be approximately twice as fast as the FC5-WENO9 hybrid. The recent contribution [1] presents a FC solver of twelfth order of accuracy. Hybridization of that solver with a WENO method, which is left for future work, is expected to give rise to higher efficiencies than the CD6-WENO hybrid. But, in any case, owing to its superior control of pollution error (see Figure 1) and general ability to handle complex, non-periodic domains at high order (as discussed in detail in the introduction sections of references $[4,5]$ ), the FC5-WENO9 method is itself expected to be significantly more efficient and flexible than CD6-WENO in applications involving general geometries 
Error in entropy amplification, Mach $=3.0, T=5.0$

\begin{tabular}{||c||c|c||c||}
\multicolumn{5}{|c||}{$N D=80, N P=33, \Delta t=6.8 \times 10^{-4}$} \\
\hline Method & Error near shock & Error far from shock & CPU time(s) \\
\hline WENO5 & $10.5 \%$ & $22.6 \%$ & 99.0 \\
FC5-WENO5 & $10.4 \%$ & $10.2 \%$ & 32.0 \\
\hline WENO9 & $4.4 \%$ & $4.0 \%$ & 188.0 \\
FC5-WENO9 & $4.4 \%$ & $4.1 \%$ & 35.0 \\
\hline
\end{tabular}

Table 1

Error in entropy amplification, Mach $=3.0, T=5.0$

$$
N D=160, N P=33, \Delta t=3.4 \times 10^{-4}
$$

\begin{tabular}{||c||c|c||c||}
\multicolumn{4}{c}{$N D=160, N P=33, \Delta t=3.4 \times 10^{-4}$} \\
\hline Method & Error near shock & Error far from shock & CPU time(s) \\
\hline WENO5 & $3.1 \%$ & $3.1 \%$ & 396.0 \\
FC5-WENO5 & $3.4 \%$ & $3.3 \%$ & 119.0 \\
\hline WENO9 & $0.7 \%$ & $0.61 \%$ & 753.0 \\
FC5-WENO9 & $0.7 \%$ & $0.6 \%$ & 126.0 \\
\hline
\end{tabular}

Table 2

Error in entropy amplification, Mach $=6.0, T=2.5$

\begin{tabular}{||c||c|c||c||}
\multicolumn{4}{c}{$N D=160, N P=33, \Delta t=2.1 \times 10^{-4}$} \\
\hline Method & Error near shock & Error far from shock & CPU time(s) \\
\hline WENO5 & $6.5 \%$ & $7.7 \%$ & 325.0 \\
FC5-WENO5 & $7.3 \%$ & $7.2 \%$ & 98.0 \\
\hline WENO9 & $1.8 \%$ & $1.7 \%$ & 618.0 \\
FC5-WENO9 & $1.8 \%$ & $1.8 \%$ & 106.0 \\
\hline
\end{tabular}

Table 3

and high frequencies in two and three dimensions - and, thus, in particular, in the treatment of general structures arising from science and engineering practice.

\subsubsection{Shock/entropy-wave interaction test}

The shock/entropy-wave interaction problem is a popular test used to evaluate the performance of high-order shock capturing schemes in the presence of 
both shocks and significant oscillatory smooth structures [14]. Owing to the coexistence of the shock discontinuities and smooth structures, this problem is also well suited for gauging the effectiveness of the multi-resolution strategy and the hybrid scheme. The spatial domain is $[-10,10]$ and the initial and boundary conditions correspond to a right-moving Mach 3 shock located at -9.5 at time $T=0.0$, and approaching a sinusoidal density wave in $[-8.85,10]$. Specifically,

$$
(\rho, v, p)(0, x)=\left\{\begin{aligned}
(3.857143,2.629369,10.33333) & x \leq-9.5 \\
(1.0,0.0,1.0) & -9.5 \leq x \leq-8.85 \\
(1.0+0.2 \sin (5(x-8.85)), 0,1.0) & x>-8.85
\end{aligned}\right.
$$

We solve the Euler system using both WENO5 and FC5-WENO5 solvers and we consider the density profiles obtained for a total number of 2561 grid points, or 40 domains of 33 points each. In Figs. 8(a)-(d), we show the solution at different times $T=0,1.25,2.5$, and 3.75, respectively. As is clear from Fig. $8(\mathrm{a})$, on the onset $(T=0)$, the multi-resolution smoothness indicator correctly identifies both the shock discontinuities at $x=-9.5$ and the discontinuities in the density derivative at $x=-8.85$. Similarly, for the later times (Figs. $8(\mathrm{c})-(\mathrm{d}))$, the scheme successfully identifies all discontinuities.

As mentioned previously, the smoothness indicator may mistakenly identify large but smooth gradients as discontinuities if insufficient resolution is used. This is evident in the case of the shock/entropy-wave interaction for long integration times, for instance at $T=5$, (Fig. 9(a)). As shown in that figure, the smooth structures in the interval $[4.0,6.0]$ are mistakenly identified as nonsmooth and treated as WENO domains. On the other hand, as shown in Fig. 9(b), increasing the resolution, from 40 domains to 80, yields a reliable shock detection strategy. It is to be noted that such increase in resolution may not be required solely for the reliable smoothness detection, but rather it may be necessary if a high level of accuracy is required. As is evident from Fig. 9(c) and (d), while the lower-resolution calculations fails to capture the small discontinuities at $x \approx 4.5$ and 5.5 (highlighted in Figures $9(\mathrm{c})$ and (d) with blue squares), the higher-resolution does resolve these small scale discontinuities.

\section{Concluding remarks}

We have presented a high-order method based on the hybridization of the Fourier continuation method and a WENO finite difference discretization for the solution of non-linear conservation laws. The hybrid strategy is based 


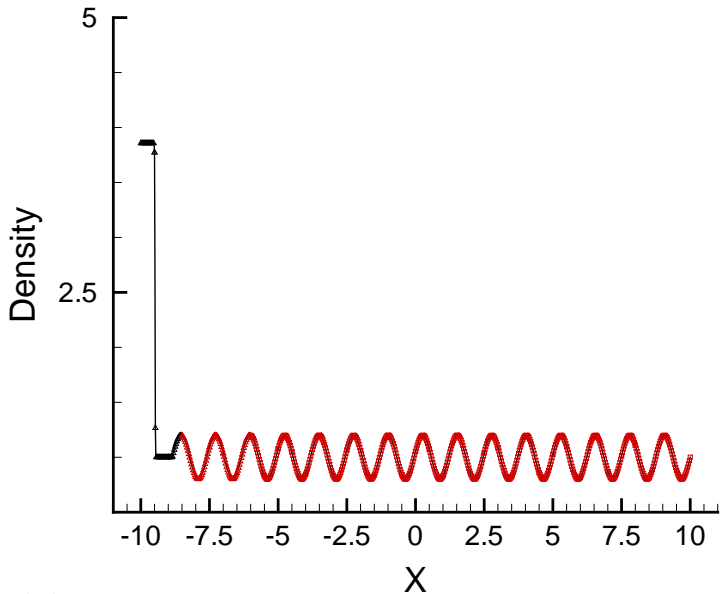

(a)

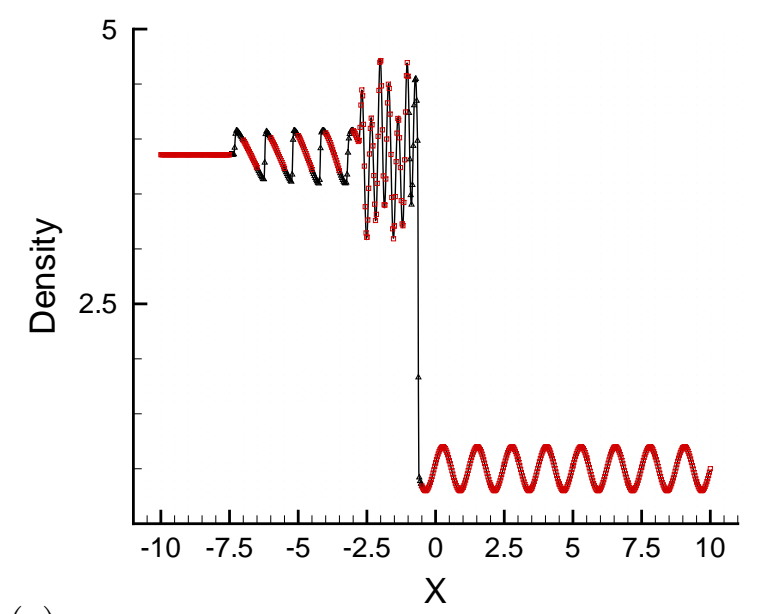

(c)

(d)

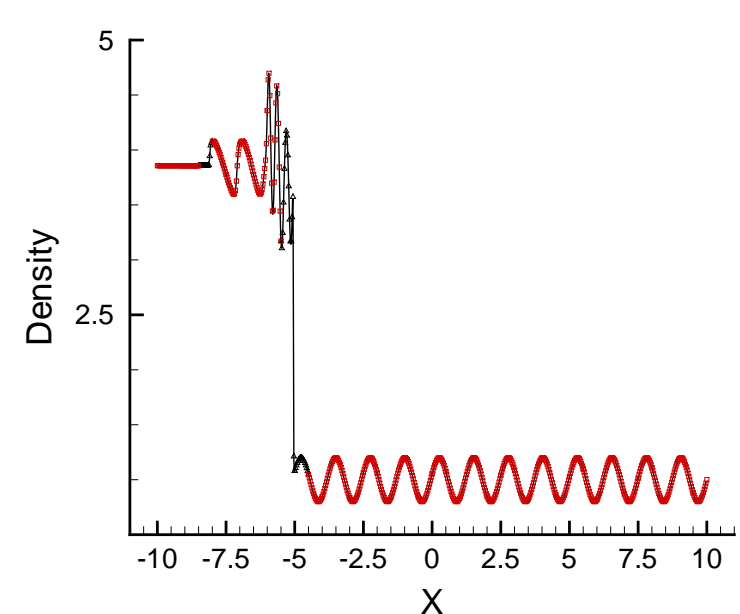

(b)

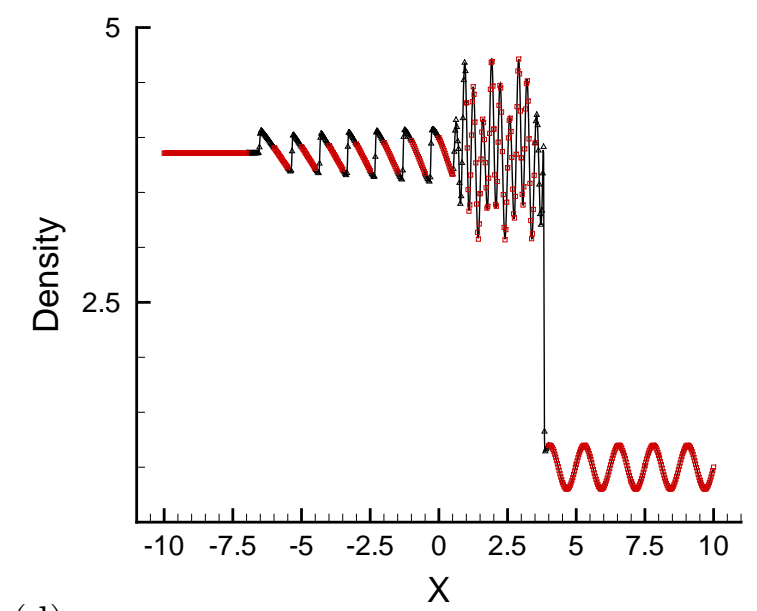

Fig. 8. Density profile for the shock-entropy wave interaction. (a) $T=0.0013$; (b) $T=1.25$; (c) $T=2.5$; (d) $T=3.75$. Solid line representing WENO5 solution with 1281 grid points, the symbols showing FC5-WENO5 solution with 40 domains of 33 grid points; $\triangle$ for the WENO domains and $\square$ for the FC domains. The location of the dotted lines signify domain boundaries in the hybrid method. The effectiveness of the multi-resolution method is clear as the the WENO domains were used only in the region of discontinuities.

on a multi-domain formulation with a smoothness detection procedure that distinctively flags the domains containing discontinuities. The domains containing discontinuities are treated by means of the WENO scheme and those with smooth solutions are discretized using the Fourier continuation method. For applications with isolated discontinuities in space and time, this strategy offers significant advantages over the pure WENO methods, since in the hybrid method the majority of the domains are discretized using the FC method which is less expensive than the WENO reconstruction, yet offers a highly efficient and accurate procedure for complex but smooth portions of the solution. 


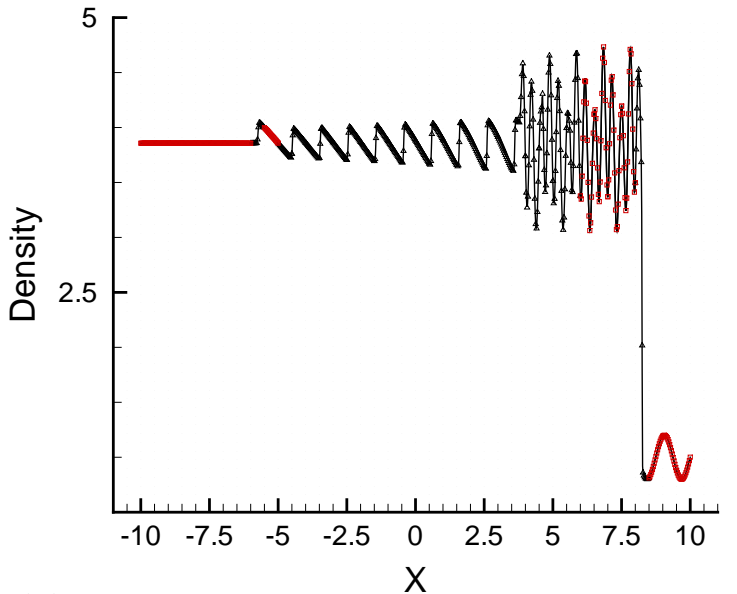

(a)

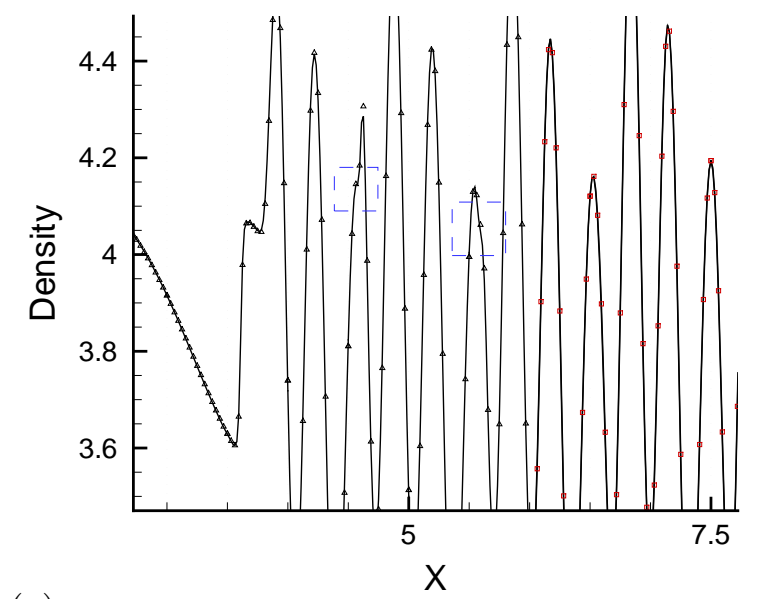

(c)

(d)

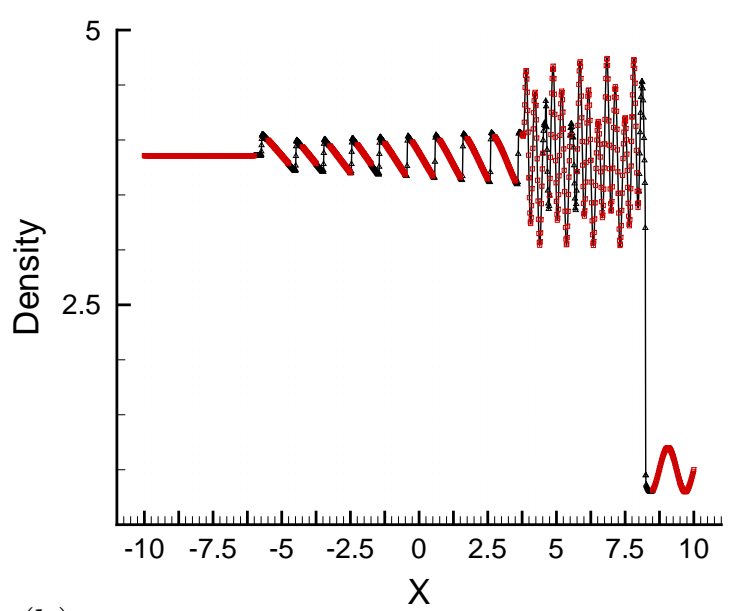

(b)

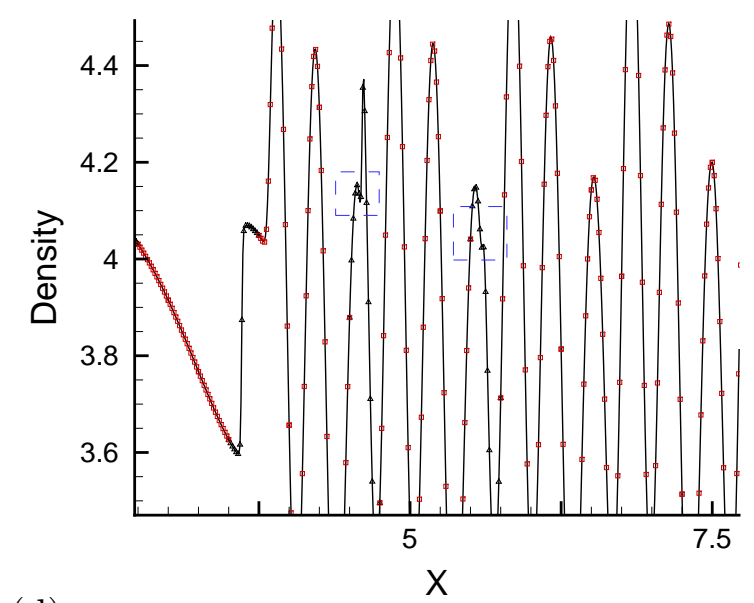

Fig. 9. Density profile for shock-entropy wave interaction for the total time of $T=5.0$; the solid line representing pure WENO5 solution, the symbols the hybrid FC5-WENO5 solution with $\triangle$ for the WENO domains and $\square$ for the FC domains. (a) Total grid points of 1281 for the WENO5 solver, 40 domains of 33 points each for the FC5-WENO5; (b) total grid points of 1601 for the WENO5 solver, 80 domains of 33 points each for the FC5-WENO5; (c) close-up view of (a) in the region of $[3.5,4.6] \times[3,7.5]$; (d) close-up view of (b) of the the same region. The location of the dotted lines signify domain boundaries. The low resolution calculations (Figs. (a), (c)) fails to capture the small discontinuities (in the blue square regions, Figs. (c)), and the multi-resolution method overestimates the number of WENO domains; the high resolution solutions (Figs. (b) and (d)) captures the small discontinuities and the multi-resolution method performs reliably.

Our hybrid FC-WENO method offers several advantages over alternative techniques based on finite difference methods or Chebyshev spectral methods. While the central difference methods suffer from the pollution error, the FC methods are pollution-error-free as is characteristic of a Fourier method. Un- 
like previous hybrid methods based on Chebyshev spectral methods, the FC method is based on an equi-spaced grid points. This not only avoids the significant cost of the extra interpolations to transfer data from the ChebyshevGauss-Lobatto points to the equi-spaced grids in the hybrid strategy, but also yields less stringent stability constraint, and thus allows for use of larger time step size in an explicit time marching scheme.

We verified the fifth-order accuracy of the FC method for smooth problems and also demonstrated the effectiveness of the exponential filter in achieving a stable FC method in the presence of the Dirichlet boundary conditions or in the multi-domain formulation. By solving the Euler system for a shock/smallentropy-wave interaction problem, we demonstrated that for a given accuracy level of $1 \%$, our hybrid strategy is several-fold faster than the pure WENO scheme and that the hybrid approach is both accurate and robust for strongly nonlinear problems. Based on these preliminary but nontrivial tests we expect that our approach will prove an enabling methodology for simulation of challenging multi-dimensional multi-scale problems such as shocked-induced transition, turbulence, and combustion - as we hope to demonstrate in future work.

\section{Acknowledgments}

This work was financially supported by US Department of Energy, under Contract DE-FG02-98ER25346. NA and OB gratefully acknowledge support from AFOSR and NSF.

\section{References}

[1] N. Albin and O. P. Bruno, A Spectral FC Solver for the Compressible Navier-Stokes Equations in General Domains I: Explicit time-stepping, To be submitted, (2010)

[2] J. Boyd, A Comparison of Numerical Algorithms for Fourier Extension of the First, Second, and Third Kinds, J. Comput. Phys. 178 (2002) 118-160.

[3] O. P. Bruno, Y. Han and M. M. Pohlman, Accurate, high-order representation of complex three-dimensional surfaces via Fourier continuation analysis, J. Comput. Phys. 227 (2007) 1094-1125.

[4] O. P. Bruno and M. Lyon, High-order unconditionally stable FC-AD solvers for general smooth domains I. Basic elements, J. Comput. Phys. 229 (2010) 2009-2033. 
[5] M. Lyon and O. P. Bruno, High-order unconditionally stable FC-AD solvers for general smooth domains II. Elliptic, parabolic and hyperbolic PDEs; theoretical considerations, J. Comput. Phys. 229 (2010) 3358-3381.

[6] L. N. Trefethen and D. Bau, III, Numerical Linear Algebra (Soc. for Industr. and Appl. Math., Philadelphia, 1997).

[7] A. Harten, Adaptive multiresolution schemes for shock computations, J. Comput. Phys. 115 (1994) 319-338.

[8] B. Costa and W. S. Don Multi-domain hybrid spectral-WENO methods for hyperbolic conservation laws, J. Comput. Phys. 224 (2007) 3358-3381.

[9] B. Costa, W. Don, D. Gottlieb and R. Sendersky, Two-Dimensional Multi-Domain Hybrid Spectral- WENO Methods for Conservation Laws, Cummunications in Comput. Physics, 1 (2006) 550-577.

[10] G.S. Jiang, C.W. Shu, Efficient implementation of weighted ENO schemes, J. Comput. Phys. 126 (1996) 202-228

[11] C.-W. Shu, Essentially non-oscillatory and weighted essentially non-oscillatory schemes for hyperbolic conservation laws, in: A. Quarteroni (Ed.), Advanced Numerical Approximation of Nonlinear Hyperbolic Equations, Lecture Notes in Mathematics, vol 1697, Springer, Cetraro, Italy, (1997) 325.

[12] C. Shu, High order weighted essentially nonoscillatory schemes for convection dominated problems, SIAM Review, 51 (2009) 82-126.

[13] D. S. Balsara and C. Shu, Monotonicity preserving weighted essentially nonoscillatory schemes with increasingly high order of accuracy, J. Comput. Phys. 160 (2000) 405-452.

[14] C.-W. Shu and S. Osher, Efficient implementation of essentially non-oscillatory shock capturing schemes, J. Comput. Phys., 77 (1988), 439-471.

[15] X-D. Liu, S. Osher, and T. Chan, Weighted Essentially Non-oscillatory Schemes, J. Comput. Phys. 115, (1994) 200-212.

[16] D. Gottlieb and J. S. Hesthaven, Spectral methods for hyperbolic problems, Journal of Computational and Applied Mathematics 128 (2001) 83-131.

[17] J. S. Hesthaven, S. Gottlieb and D. Gottlieb, Spectral Methods for TimeDependent Problems, Cambridge Monographs on Applied and Computational Mathematics 21. Cambridge University Press, Cambridge, UK. X+274 pages, January 2007.

[18] M. K. Morkovin, Note on the assessment of flow disturbances at a blunt body traveling at supersonic speeds owing to flow disturbance in free stream. J. Applied Mechanics 27 (1960) 223-229.

[19] J. Shi, Y. Zhang and C. Shu, Resolution of high order WENO schemes for complicated flow structures, J. Comput. Phys. 186 (2003) 690-696. 
[20] M. Latini, O. Schilling and W. Don, Effects of WENO flux reconstruction order and spatial resolution on reshocked two-dimensional Richtmyer-Meshkov instability, J. Comput. Phys. 221 (2007) 805-836.

[21] O. Schilling and M. Latini, High-order WENO simulations of three-dimensional reshocked Richtmyer-Meshkov instability to late times: dynamics, dependence on initial conditions, and comparisons to experimental data, Acta Mathematica Scientia 30 (2010), 595-620.

[22] I.A. Babuska and S.A. Sauter. Is the pollution the FEM avoidable for the Helmholtz equation considering high wave numbers? SIAM J. Numer. Anal., 34 (1997) 2392-2423.

[23] L. Jameson. High order schemes for resolving waves: Number of points per wavelength. J. Sci. Comput., 15 (2000) 417-433.

[24] S. Gottlieb and C. Shu, Total variation diminishing Runge-Kutta schemes, Math. Comp. 67 (1998), 73-85.

[25] G. Dahlquist and A. Bjork Numerical Methods, Prentice Hall, Englewood Clis, 1974.

[26] B. Gustafsson, High Order Difference Methods for Time Dependent PDE, Springer, 2008. 